\title{
Entanglement-assisted transformation is asymptotically equivalent to multiple-copy transformation
}

\author{
Runyao Duan, , 因 Yuan Feng, , 团 and Mingsheng Ying, 1 , \\ ${ }^{1}$ State Key Laboratory of Intelligent Technology and Systems, \\ Department of Computer Science and Technology, Tsinghua University, Beijing, China, 100084
}

(Dated: September 12, 2018)

\begin{abstract}
We show that two ways of manipulation of quantum entanglement, namely, entanglement-assisted local transformation [D. Jonathan and M. B. Plenio, Phys. Rev. Lett. 83, 3566 (1999)] and multiplecopy transformation [S. Bandyopadhyay, V. Roychowdhury, and U. Sen, Phys. Rev. A 65, 052315 (2002)], are equivalent in the sense that they can asymptotically simulate each other's ability to implement a desired transformation from a given source state to another given target state with the same optimal success probability. As a consequence, this yields a feasible method to evaluate the optimal conversion probability of an entanglement-assisted transformation.

PACS numbers: 03.67.Hk, 03.67.Mn
\end{abstract}

As a valuable resource in quantum information processing, quantum entanglement has been widely used in quantum cryptography [1], quantum superdense coding [2], and quantum teleportation [3]. Consequently, it remains the subject of interest at present after years of investigations.

Unlike common resources, it was discovered by Jonathan and Plenio [4] that quantum entanglement is truly a strange one: sometimes it can help quantum information processing without being consumed at all. This effect can be understood in the situation of entanglement transformation. Suppose that two spatially separated parties, say, Alice and Bob, share a finite dimensional entangled pure state $\left|\psi_{1}\right\rangle$, and they want to convert $\left|\psi_{1}\right\rangle$ into another state $\left|\psi_{2}\right\rangle$, by using local quantum operations and classical communication (LOCC) only [4, 5, 6, 7, 8, 9, 10, 11, 12, 13, 14, 16, 17]. For certain $\left|\psi_{1}\right\rangle$ and $\left|\psi_{2}\right\rangle$, they can accomplish their goal with certainty by constructing a local protocol [ $]$ ]. While in general, only a maximal conversion probability less than one can be achieved [7]. In the latter case, Jonathan and Plenio demonstrated by examples that sometimes Alice and Bob may borrow another entangled state $|\phi\rangle$, known as a catalyst, to realize the transformation $\left|\psi_{1}\right\rangle \rightarrow\left|\psi_{2}\right\rangle$ with probability one. The transformation can be represented as $\left|\psi_{1}\right\rangle \otimes|\phi\rangle \rightarrow\left|\psi_{2}\right\rangle \otimes|\phi\rangle$, in which it is obvious that the catalyst $|\phi\rangle$ is not consumed during the process. Such a transformation that uses intermediate entanglement without consuming it is called 'entanglement-assisted local transformation' in Ref. [4], abbreviated to ELOCC. The mathematical structure of ELOCC has been studied thoroughly in Refs. 9, 10, 11. It has also been shown that such an entanglement catalysis phenomenon exists in the manipulation of mixed states [12], and in the implementation of non-local quantum operations [13].

Another interesting way of manipulating quantum en-

\footnotetext{
*Electronic address: dry02@mails.tsinghua.edu.cn

${ }^{\dagger}$ Electronic address: feng-y@tsinghua.edu.cn

${ }^{\ddagger}$ Electronic address: yingmsh@tsinghua.edu.cn
}

tanglement was proposed by Bandyopadhyay et al [14]. Specifically, they found that sometimes multiple copies of source state may be transformed into the same number of target state although the transformation cannot happen for a single copy. That is, for some states $\left|\psi_{1}\right\rangle$ and $\left|\psi_{2}\right\rangle$, although Alice and Bob cannot transform $\left|\psi_{1}\right\rangle$ into $\left|\psi_{2}\right\rangle$ with certainty by LOCC, there may exist $m>1$ such that they can realize the transformation $\left|\psi_{1}\right\rangle^{\otimes m} \rightarrow\left|\psi_{2}\right\rangle^{\otimes m}$ with certainty. This kind of transformation that uses multiple copies of source state and then transforms all of them together into the same number of target state is called 'non-asymptotic bipartite pure-state entanglement transformation' in [14]. More intuitively, it can also be called 'multiple-copy entanglement transformation', or MLOCC for short [15]. The mathematical structure of MLOCC was carefully examined in Ref. [16].

At first glance, entanglement-assisted transformation and multiple-copy entanglement transformation are two completely different extensions of ordinary LOCC. To achieve a specific transformation, the former needs to borrow extra entanglement as resource but is promised not to consume it during the transformation, while the latter realizes a similar purpose by accumulating a sufficiently large number of copies of source state and then transforms all these copies together into the same number of target state.

A surprising fact is that these two kind of manipulations of entanglement are closely related to each other. In Ref. [16] it was demonstrated that if a bipartite entangled state $\left|\psi_{1}\right\rangle$ can be transformed into another bipartite entangled state $\left|\psi_{2}\right\rangle$ with certainty by using MLOCC, then $\left|\psi_{1}\right\rangle$ can also be deterministically transformed into $\left|\psi_{2}\right\rangle$ by using a catalyst. In other words, in the deterministic scenario ELOCC is at least as powerful as MLOCC. However, this result is of limited interest because in general for any two given states $\left|\psi_{1}\right\rangle$ and $\left|\psi_{2}\right\rangle$ the transformation of $\left|\psi_{1}\right\rangle$ to $\left|\psi_{2}\right\rangle$ cannot be achieved with certainty by using ELOCC [4]. It is a very interesting problem to further explore the precise relation between ELOCC and MLOCC.

An interesting equivalence of ELOCC and MLOCC 
was observed by the authors previously in Ref. [17], where we only concerned that whether ELOCC or MLOCC transformations have advantages over pure LOCC ones in producing a given target. Specifically, for an $n \times n$ target state $\left|\psi_{2}\right\rangle$ and a positive integer $k$, if there exists another $n \times n$ state $\left|\psi_{1}\right\rangle$ such that the transformation of $\left|\psi_{1}\right\rangle$ to $\left|\psi_{2}\right\rangle$ can be achieved with certainty by using a $k \times k$-dimensional catalyst while $\left|\psi_{1}\right\rangle$ cannot be transformed into $\left|\psi_{2}\right\rangle$ by LOCC, then we say $k$-ELOCC is useful in producing $\left|\psi_{2}\right\rangle$. The concept that $k$-MLOCC is useful in producing a given target state can be defined in a similar way. Then we proved that $k$-ELOCC is useful in producing $\left|\psi_{2}\right\rangle$ if and only if $k$-MLOCC is useful in producing the same target. An explicit necessary and sufficient condition for both of them in terms of Schmidt coefficients of $\left|\psi_{2}\right\rangle$ was also obtained. This equivalence was then generalized to probabilistic transformations. See Theorems 2 and 4 in Ref. 17] for details. Obviously, this kind of equivalence is weak in the sense that only the target state is involved, and the source state is irrelevant.

In this brief report, we consider probabilistic transformations instead of deterministic ones and we obtain a very strong equivalence between ELOCC and MLOCC. We find that ELOCC and MLOCC are indeed equivalent in the sense that they can simulate each other's ability to implement any given transformation with the same optimal success probability. More precisely, let $\left|\psi_{1}\right\rangle$ and $\left|\psi_{2}\right\rangle$ be the source state and the target state of a transformation, respectively. We further assume that $\left.P_{E}\left(\left|\psi_{1} \rightarrow\right| \psi_{2}\right\rangle\right)$ (see Eq. (44) bellow) is the optimal success probability that can be achieved by using some catalyst. Similarly, $P_{M}\left(\psi_{1} \rightarrow\left|\psi_{2}\right\rangle\right)$ (see Eq. (3) bellow) is the optimal average probability that can be achieved by transforming multiple copies of $\left|\psi_{1}\right\rangle$ into the same number of copies of $\left|\psi_{2}\right\rangle$. Then we prove that $\left.P_{E}\left(\left|\psi_{1} \rightarrow\right| \psi_{2}\right\rangle\right)$ is exactly the same as $\left.P_{M}\left(\left|\psi_{1} \rightarrow\right| \psi_{2}\right\rangle\right)$ for any choices of $\left|\psi_{1}\right\rangle$ and $\left|\psi_{2}\right\rangle$. It is clear that this equivalence is very different from the one obtained in Ref. [17]. In fact it is much more elaborate since it completely characterizes the equivalence of ELOCC and MLOCC in the probabilistic scenario.

This equivalence of ELOCC and MLOCC transformations is interesting in many ways, both theoretically and practically. In principle, it uncovers an essential connection between entanglement catalysis and multiplecopy entanglement transformation, and declares that they have almost the same effect. In practice, it provides a more feasible way to evaluate the optimal conversion probability of an ELOCC transformation by calculating the optimal conversion probability of the corresponding MLOCC one. The proof presented in this brief report also reveals that such an equivalence is deeply related to a well-known fact: a maximally entangled state cannot serve as a catalyst, which puts a fundamental constraint on the power of entanglement-assisted transformation.

Let us begin with a concrete example to examine the relationship between entanglement-assisted transformation and multiple-copy entanglement transforma- tion. The primary tool required for this is Vidal's formula [7]. Let $\left|\psi_{1}\right\rangle=\sum_{i=1}^{n} \sqrt{\alpha_{i}}\left|i_{A}\right\rangle\left|i_{B}\right\rangle$ and $\left|\psi_{2}\right\rangle=$ $\sum_{i=1}^{n} \sqrt{\beta_{i}}\left|i_{A}\right\rangle\left|i_{B}\right\rangle$ be pure bipartite entangled states with ordered Schmidt coefficients $\alpha_{1} \geq \alpha_{2} \geq \cdots \geq \alpha_{n}>0$ and $\beta_{1} \geq \beta_{2} \geq \cdots \geq \beta_{n} \geq 0$, respectively. Then the maximal conversion probability of transforming $\left|\psi_{1}\right\rangle$ into $\left|\psi_{2}\right\rangle$ by LOCC is given by [7]

$$
p_{\max }\left(\left|\psi_{1}\right\rangle \rightarrow\left|\psi_{2}\right\rangle\right)=\min \left\{\frac{E_{l}\left(\left|\psi_{1}\right\rangle\right)}{E_{l}\left(\left|\psi_{2}\right\rangle\right)}: 1 \leq l \leq n\right\},
$$

where $E_{l}\left(\left|\psi_{1}\right\rangle\right)=\sum_{i=l}^{n} \alpha_{i}$. In the case of $p_{\max }\left(\left|\psi_{1}\right\rangle \rightarrow\right.$ $\left.\left|\psi_{2}\right\rangle\right)=1$, i.e., the transformation $\left|\psi_{1}\right\rangle \rightarrow\left|\psi_{2}\right\rangle$ can be realized with certainty under LOCC, Vidal's formula reduces to Nielsen's theorem [6].

Now an example demonstrating the power of entanglement-assisted transformation is as follows. Take $\left|\psi_{1}\right\rangle=\sqrt{0.4}|00\rangle+\sqrt{0.4}|11\rangle+\sqrt{0.1}|22\rangle+\sqrt{0.1}|33\rangle$ and $\left|\psi_{2}\right\rangle=\sqrt{0.5}|00\rangle+\sqrt{0.25}|11\rangle+\sqrt{0.25}|22\rangle$. We know that the transformation $\left|\psi_{1}\right\rangle \rightarrow\left|\psi_{2}\right\rangle$ cannot occur with certainty under LOCC since $p_{\max }\left(\left|\psi_{1}\right\rangle \rightarrow\left|\psi_{2}\right\rangle\right)=0.8$. But if another entangled state $|\phi\rangle=\sqrt{0.6}|44\rangle+\sqrt{0.4}|55\rangle$ is introduced, then the transformation $\left|\psi_{1}\right\rangle \otimes|\phi\rangle \rightarrow\left|\psi_{2}\right\rangle \otimes|\phi\rangle$ can be realized with certainty because $p_{\max }\left(\left|\psi_{1}\right\rangle \otimes|\phi\rangle \rightarrow\right.$ $\left.\left|\psi_{2}\right\rangle \otimes|\phi\rangle\right)=1$. Interestingly, the same task can be achieved by a multiple-copy entanglement transformation. It is not difficult to see that the transformation $\left|\psi_{1}\right\rangle^{\otimes 3} \rightarrow\left|\psi_{2}\right\rangle^{\otimes 3}$ occurs with certainty by checking $p_{\max }\left(\left|\psi_{1}\right\rangle^{\otimes 3} \rightarrow\left|\psi_{2}\right\rangle^{\otimes 3}\right)=1$. That is, when Alice and Bob prepare three copies of $\left|\psi_{1}\right\rangle$ instead of just a single one, they can transform these three copies all together into three copies of $\left|\psi_{2}\right\rangle$ by LOCC.

In the above example, entanglement-assisted transformation and multiple-copy entanglement transformation can be simulated by each other. Indeed, it is not difficult to find more examples in which the same thing happens. This motivates us to conjecture that these two kinds of manipulation of entanglement are in fact equivalent in the sense that they can be simulated by each other in some way.

As will be seen later, every multiple-copy entanglement transformation can be realized by an entanglementassisted one. Unfortunately, the following example shows that sometimes an entanglement-assisted transformation is more powerful than a corresponding multiplecopy entanglement transformation. Let us take source state and target state as $\left|\psi_{1}\right\rangle=\frac{1}{\sqrt{1.01}}(\sqrt{0.40}|00\rangle+$ $\sqrt{0.40}|11\rangle+\sqrt{0.10}|22\rangle+\sqrt{0.1}|33\rangle+\sqrt{0.01}|44\rangle)$ and $\left|\psi_{2}\right\rangle=$ $\frac{1}{\sqrt{1.01}}(\sqrt{0.50}|00\rangle+\sqrt{0.25}|11\rangle+\sqrt{0.20}|22\rangle+\sqrt{0.05}|33\rangle+$ $\sqrt{0.01}|44\rangle)$, respectively. A simple calculation carries out that $|\phi\rangle$ given in the above example is a multiple-copy catalyst for the transformation from $\left|\psi_{1}\right\rangle$ to $\left|\psi_{2}\right\rangle$, since it holds that $p_{\max }\left(\left|\psi_{1}\right\rangle \otimes|\phi\rangle^{\otimes 11} \rightarrow\left|\psi_{2}\right\rangle \otimes|\phi\rangle^{\otimes 11}\right)=1$. On the other hand, noticing here that the least Schmidt coefficients of the source and target are very small and identical, we can show that $p_{\max }\left(\left|\psi_{1}\right\rangle^{\otimes m} \rightarrow\left|\psi_{2}\right\rangle^{\otimes m}\right)<$ 1 for any $m \geq 1$ by a tedious but routine calculation. Therefore, for all $m \geq 1$, it always holds that $p_{\max }\left(\left|\psi_{1}\right\rangle^{\otimes m} \rightarrow\left|\psi_{2}\right\rangle^{\otimes m}\right)<p_{\max }\left(\left|\psi_{1}\right\rangle \otimes|\phi\rangle^{\otimes 11} \rightarrow\left|\psi_{2}\right\rangle \otimes\right.$ 
$\left.|\phi\rangle^{\otimes 11}\right)$. Such an example shows that an entanglementassisted transformation cannot be realized by a multiplecopy entanglement transformation in a finite manner.

We now turn to observe the relationship between entanglement-assisted transformation and multiple-copy entanglement transformation in an asymptotical manner. Surprisingly, these two kinds of transformation are asymptotically equivalent although it is not the case when only a finite manner is allowed. To verify this, we need to introduce several notations. For each $m \geq 1$, let

$$
p_{M}^{(m)}\left(\left|\psi_{1}\right\rangle \rightarrow\left|\psi_{2}\right\rangle\right)=\left[p_{\max }\left(\left|\psi_{1}\right\rangle^{\otimes m} \rightarrow\left|\psi_{2}\right\rangle^{\otimes m}\right)\right]^{\frac{1}{m}} .
$$

Intuitively, $p_{M}^{(m)}$ is the geometric average value of the probability of (single-copy) transformation $\left|\psi_{1}\right\rangle \rightarrow\left|\psi_{2}\right\rangle$ when considering in the environment of $m$-copy transformation $\left|\psi_{1}\right\rangle^{\otimes m} \rightarrow\left|\psi_{2}\right\rangle^{\otimes m}$. (Note that it is reasonable to compare the probability $p_{\max }\left(\left|\psi_{1}\right\rangle \otimes|\phi\rangle \rightarrow\left|\psi_{2}\right\rangle \otimes|\phi\rangle\right)$ with $p_{M}^{(m)}\left(\left|\psi_{1}\right\rangle \rightarrow\left|\psi_{2}\right\rangle\right)$ rather than $p_{\max }\left(\left|\psi_{1}\right\rangle^{\otimes m} \rightarrow\right.$ $\left.\left|\psi_{2}\right\rangle^{\otimes m}\right)$, because the latter is the probability that $m$ copies of $\left|\psi_{1}\right\rangle$ are transformed simultaneously to the same number of $\left|\psi_{2}\right\rangle$ and it is usually the $m$ th power of the probability of single-copy transformation.) Then the optimal conversion probability of a multiple-copy entanglement transformation is given by

$$
P_{M}\left(\left|\psi_{1}\right\rangle \rightarrow\left|\psi_{2}\right\rangle\right)=\sup _{m} p_{M}^{(m)}\left(\left|\psi_{1}\right\rangle \rightarrow\left|\psi_{2}\right\rangle\right),
$$

where $m$ ranges over all positive integers. On the other hand, we define the optimal conversion probability of an entanglement-assisted transformation from $\left|\psi_{1}\right\rangle$ to $\left|\psi_{2}\right\rangle$ by

$$
P_{E}\left(\left|\psi_{1}\right\rangle \rightarrow\left|\psi_{2}\right\rangle\right)=\sup _{|\phi\rangle} p_{\max }\left(\left|\psi_{1}\right\rangle \otimes|\phi\rangle \rightarrow\left|\psi_{2}\right\rangle \otimes|\phi\rangle\right),
$$

where $|\phi\rangle$ ranges over all finite dimensional bipartite pure states.

Now with the notations introduced above, the asymptotical equivalence of ELOCC and MLOCC can be exactly stated in the following:

Theorem 1 For any pure bipartite states $\left|\psi_{1}\right\rangle$ and $\left|\psi_{2}\right\rangle$, $P_{E}\left(\left|\psi_{1}\right\rangle \rightarrow\left|\psi_{2}\right\rangle\right)=P_{M}\left(\left|\psi_{1}\right\rangle \rightarrow\left|\psi_{2}\right\rangle\right)$.

Proof. For simplicity, in this proof we abbreviate $P_{E}\left(\left|\psi_{1}\right\rangle \rightarrow\left|\psi_{2}\right\rangle\right)$ and $P_{M}\left(\left|\psi_{1}\right\rangle \rightarrow\left|\psi_{2}\right\rangle\right)$ to $P_{E}$ and $P_{M}$, respectively.

We first prove that $P_{E} \geq P_{M}$. In fact, we can prove that any multiple-copy entanglement transformation can be simulated by a suitable entanglement-assisted transformation with a finite dimensional catalyst state.

For simplicity of notations, for any $m \geq 1$, we denote the geometric average probability of $m$-copy transformation, namely $p_{M}^{(m)}\left(\left|\psi_{1}\right\rangle \rightarrow\left|\psi_{2}\right\rangle\right)$ in Eq. (2), by $p_{m}$. We will show that there always exists a finite dimensional catalyst state $|\phi\rangle$ such that

$$
p_{\max }\left(\left|\psi_{1}\right\rangle \otimes|\phi\rangle \rightarrow\left|\psi_{2}\right\rangle \otimes|\phi\rangle\right) \geq p_{m} .
$$

Then $P_{E} \geq P_{M}$ follows immediately from Eq. (5), by taking supremums according to $m$ and $|\phi\rangle$, respectively.

For this purpose, let us construct a catalyst state $|\phi\rangle$ as follows:

$$
\lambda(\phi)=\lambda\left(\psi_{1}^{\otimes m-1} \oplus p_{m} \psi_{1}^{\otimes m-2} \otimes \psi_{2} \oplus \cdots \oplus p_{m}^{m-1} \psi_{2}^{\otimes m-1}\right),
$$

where we use $\lambda(\phi)$ to denote the ordered Schmidt coefficient vector of $|\phi\rangle$ and an unimportant normalization factor of $\lambda(\phi)$ is omitted. The intuition behind such a construction comes from the following well known algebraic identity:

$x^{m}-p^{m} y^{m}=(x-p y)\left(x^{m-1}+p x^{m-2} y+\cdots+p^{m-1} y^{m-1}\right)$.

Now by using Lemmas 3 and 4 in Ref. 10] repeatedly, we can easily verify the validity of Eq. (5). Thus we complete the proof of $P_{E} \geq P_{M}$.

Conversely, we prove the other part that $P_{E} \leq P_{M}$. To this end, a natural strategy is to show that the role of a catalyst state $|\phi\rangle$ can always be replaced by a suitable $m$-copy transformation. Unfortunately, according to the second example presented above, this idea does not work. To overcome this difficulty, we try to simulate a catalyst state by using multiple-copy entanglement transformation in an asymptotical manner.

Before going into the detailed proof, we describe some basic proof ideas here. Two points are crucial in the subsequent proof: the first one is that we can always simulate any $k \times k$ state $|\phi\rangle$ by a maximally entangled state $\left|\Phi_{k}\right\rangle=\frac{1}{\sqrt{k}} \sum_{i=1}^{k}\left|i_{A}\right\rangle\left|i_{B}\right\rangle$ with a nonzero probability; the second one is that a maximally entangled state does not have catalysis effect, which puts a fundamental constraint on the power of entanglement catalysis.

Let us continue the proof of $P_{E} \leq P_{M}$. Assume that $|\phi\rangle$ is a $k \times k$ catalyst with the least Schmidt coefficient $\gamma_{k}>0$. To generate such a state, Alice and Bob first borrow a maximally entangled state $\left|\Phi_{k}\right\rangle$. Then they try to obtain a lower bound of the successful conversion probability of the transformation from $\left|\psi_{1}\right\rangle^{\otimes m} \otimes\left|\Phi_{k}\right\rangle$ to $\left|\psi_{2}\right\rangle^{\otimes m} \otimes\left|\Phi_{k}\right\rangle$. A possible protocol implementing this task consists of the following three steps:

1) Generate catalyst state $|\phi\rangle$. That is, perform the transformation

$$
\left|\psi_{1}\right\rangle^{\otimes m} \otimes\left|\Phi_{k}\right\rangle \rightarrow\left|\psi_{1}\right\rangle^{\otimes m} \otimes|\phi\rangle .
$$

The maximal successful conversion probability is denoted by $p_{1}$. This transformation can be realized by transforming $\left|\Phi_{k}\right\rangle$ directly into $|\phi\rangle$ and keeping $\left|\psi_{1}\right\rangle^{\otimes m}$ intact. Thus

$$
p_{1}=p_{\max }\left(\left|\Phi_{k}\right\rangle \rightarrow|\phi\rangle\right)=1 .
$$

2) Catalyze $\left|\psi_{1}\right\rangle^{\otimes m}$ into $\left|\psi_{2}\right\rangle^{\otimes m}$. That is, perform the transformation

$$
\left|\psi_{1}\right\rangle^{\otimes m} \otimes|\phi\rangle \rightarrow\left|\psi_{2}\right\rangle^{\otimes m} \otimes|\phi\rangle .
$$

The maximal successful conversion probability is denoted by $p_{2}$. This transformation can be achieved by repeatedly 
transforming $\left|\psi_{1}\right\rangle$ into $\left|\psi_{2}\right\rangle m$ times with $|\phi\rangle$ serving as a catalyst. Note that the key point here is that as a catalyst, $|\phi\rangle$ here, is reusable. Thus

$$
p_{2} \geq\left[p_{\max }\left(\left|\psi_{1}\right\rangle \otimes|\phi\rangle \rightarrow\left|\psi_{2}\right\rangle \otimes|\phi\rangle\right)\right]^{m} .
$$

3) Return the maximally entangled state $\left|\Phi_{k}\right\rangle$. That is, perform the transformation

$$
\left|\psi_{2}\right\rangle^{\otimes m} \otimes|\phi\rangle \rightarrow\left|\psi_{2}\right\rangle^{\otimes m} \otimes\left|\Phi_{k}\right\rangle .
$$

The maximal successful conversion probability is denoted by $p_{3}$. It is easy to see that this transformation can be implemented by transforming $|\phi\rangle$ into $\left|\Phi_{k}\right\rangle$ and keeping $\left|\psi_{2}\right\rangle^{\otimes m}$ intact. Thus

$$
p_{3} \geq p_{\max }\left(|\phi\rangle \rightarrow\left|\Phi_{k}\right\rangle\right)=k \gamma_{k}
$$

The above three steps complete a concrete protocol to realize the transformation from $\left|\psi_{1}\right\rangle^{\otimes m} \otimes\left|\Phi_{k}\right\rangle$ to $\left|\psi_{2}\right\rangle^{\otimes m} \otimes\left|\Phi_{k}\right\rangle$. Hence we obtain a lower bound for the maximal successful conversion probability of this transformation, that is,

$$
p_{\max }\left(\left|\psi_{1}\right\rangle^{\otimes m} \otimes\left|\Phi_{k}\right\rangle \rightarrow\left|\psi_{2}\right\rangle^{\otimes m} \otimes\left|\Phi_{k}\right\rangle\right) \geq p_{1} p_{2} p_{3}
$$

As we just mentioned above, a maximally entangled state cannot be used to catalyze any transformation, which can be treated as a direct consequence of Vidal's formula. Thus,

$$
\begin{aligned}
& p_{\max }\left(\left|\psi_{1}\right\rangle^{\otimes m} \otimes\left|\Phi_{k}\right\rangle \rightarrow\left|\psi_{2}\right\rangle^{\otimes m} \otimes\left|\Phi_{k}\right\rangle\right) \\
& =p_{\max }\left(\left|\psi_{1}\right\rangle^{\otimes m} \rightarrow\left|\psi_{2}\right\rangle^{\otimes m}\right) \text { for any } m \geq 1 .
\end{aligned}
$$

Combining Eqs. (10) and (11), we finally have

$$
p_{\max }\left(\left|\psi_{1}\right\rangle^{\otimes m} \rightarrow\left|\psi_{2}\right\rangle^{\otimes m}\right) \geq p_{1} p_{2} p_{3},
$$

Substituting Eqs. (7), (8) and (9) into Eq. (12) and taking average yield that

$$
\begin{aligned}
& {\left[p_{\max }\left(\left|\psi_{1}\right\rangle^{\otimes m} \rightarrow\left|\psi_{2}\right\rangle^{\otimes m}\right)\right]^{\frac{1}{m}}} \\
& \geq\left(k \gamma_{k}\right)^{\frac{1}{m}} p_{\max }\left(\left|\psi_{1}\right\rangle \otimes|\phi\rangle \rightarrow\left|\psi_{2}\right\rangle \otimes|\phi\rangle\right) .
\end{aligned}
$$

The above equation has an interesting physical meaning: the success probability of simulating a catalyst $|\phi\rangle$ by an $m$-copy transformation has a lower bound $\left(k \gamma_{k}\right)^{\frac{1}{m}}$. Taking supremum according to $m$ yields

$$
P_{M} \geq p_{\max }\left(\left|\psi_{1}\right\rangle \otimes|\phi\rangle \rightarrow\left|\psi_{2}\right\rangle \otimes|\phi\rangle\right) .
$$

Here we have used a well known result in elementary calculus:

$$
\sup _{m} a^{\frac{1}{m}}=1, \text { for any } 0<a \leq 1
$$

where the supremum 1 can be attained asymptotically when $m$ tends to infinity. This fact will be useful latter.

We thus finish the simulation of the catalyst state $|\phi\rangle$ by means of MLOCC asymptotically. Now the desired result $P_{E} \leq P_{M}$ follows directly by taking supremum according to $|\phi\rangle$ in the right-hand side of Eq. (14).

As we mentioned above, a direct application of Theorem 1 is to evaluate the optimal conversion probability of an ELOCC transformation. To be specific, let $\left|\psi_{1}\right\rangle$ and $\left|\psi_{2}\right\rangle$ be two given $n \times n$ states. First, we show that the geometric average conversion probability of $m$ copy transformation $\left|\psi_{1}\right\rangle^{\otimes m} \rightarrow\left|\psi_{2}\right\rangle^{\otimes m}$, or shortly, $P_{M}^{(m)}$, can be computed within the polynomial time of $m$ when $n$ is fixed. It is easy to check that both $\left|\psi_{1}\right\rangle^{\otimes m}$ and $\left|\psi_{2}\right\rangle^{\otimes m}$ have at most $\left(\begin{array}{c}n-1+m \\ n-1\end{array}\right)$ distinct Schmidt coefficients. Then one only needs $O\left(\left(\begin{array}{c}n-1+m \\ n-1\end{array}\right) \log \left(\begin{array}{c}n-1+m \\ n-1\end{array}\right)\right)$ time to sort the Schmidt coefficients of $\left|\psi_{1}\right\rangle^{\otimes m}$ and $\left|\psi_{2}\right\rangle^{\otimes m}$ into nonincreasing order, respectively. By Vidal's formula, the geometric average conversion probability of $m$-copy transformation can be calculated efficiently. A more careful analysis shows that the time complexity is about $O\left(\left(\begin{array}{c}n-1+m \\ n-1\end{array}\right) \log \left(\begin{array}{c}n-1+m \\ n-1\end{array}\right)\right)$, which is essentially the same as $O\left(m^{n-1} \log m\right)$ when $n$ is fixed. Second, by Eq. (3), we can obtain an approximation of $P_{M}$ as accurate as possible by calculating $P_{M}^{(m)}$ for a large $m$ since $P_{M}^{(m)}$ converges to $P_{M}$ when $m$ tends to infinity (this can be seen from Eq. (15) and the line after it). Third, by Theorem 11. such an approximation of $P_{M}$ can also be used to approximate $P_{E}$.

In the proof of Theorem 11 we notice that any multiple-copy transformation can be simulated by an entanglement-assisted transformation in a finite manner. However, the proof of the converse part, i.e., simulating ELOCC by MLOCC, is only given in an asymptotical way. Thereby a natural question is to ask whether one can design a stronger protocol which can simulate an entanglement-assisted transformation with a finite dimensional catalyst by using a multiple-copy entanglement transformation in a finite manner. The second example proposed above indicates that such a finite simulation is not possible for certain catalyst state. Interestingly, except for a special case, any finite dimensional catalyst can be simulated by a multiple-copy transformation in a finite manner.

Theorem 2 For any transformation $\left|\psi_{1}\right\rangle \rightarrow\left|\psi_{2}\right\rangle$ and a catalyst $|\phi\rangle$, denote $p=p_{\max }\left(\left|\psi_{1}\right\rangle \otimes|\phi\rangle \rightarrow\left|\psi_{2}\right\rangle \otimes|\phi\rangle\right)$. If $p<\min \left\{1, \frac{\alpha_{n}}{\beta_{n}}\right\}$, then there exists a finite positive integer $m$ such that $p_{M}^{(m)}\left(\left|\psi_{1}\right\rangle \rightarrow\left|\psi_{2}\right\rangle\right) \geq p$, where $\alpha_{n}$ and $\beta_{n}$ denote the least Schmidt coefficients of $\left|\psi_{1}\right\rangle$ and $\left|\psi_{2}\right\rangle$, respectively.

In other words, the geometric average value of the probability of (single-copy) transformation $\left|\psi_{1}\right\rangle \rightarrow\left|\psi_{2}\right\rangle$ in the environment of $m$-copy transformation $\left|\psi_{1}\right\rangle^{\otimes m} \rightarrow$ $\left|\psi_{2}\right\rangle^{\otimes m}$ is not less than $p$. This means that under the assumption, the role of the catalyst $|\phi\rangle$ can be replaced by an $m$-copy transformation.

Proof. First, notice that Theorem 5 in Ref. [10] provides a necessary and sufficient condition of when ELOCC is more powerful than mere LOCC. Applying this result to the transformation $\left|\psi_{1}\right\rangle \otimes|\phi\rangle \rightarrow\left|\psi_{2}\right\rangle \otimes|\phi\rangle$ 
yields

$P_{E}\left(\left|\psi_{1}\right\rangle \otimes|\phi\rangle \rightarrow\left|\psi_{2}\right\rangle \otimes|\phi\rangle\right)>p$ iff $p<\min \left\{1, \frac{\alpha_{n} \gamma_{k}}{\beta_{n} \gamma_{k}}\right\}$,

where $\gamma_{k}$ is the least Schmidt coefficient of $|\phi\rangle$.

Second, by the definition of $P_{E}\left(\left|\psi_{1}\right\rangle \rightarrow\left|\psi_{2}\right\rangle\right)$, we have

$$
P_{E}\left(\left|\psi_{1}\right\rangle \rightarrow\left|\psi_{2}\right\rangle\right)=P_{E}\left(\left|\psi_{1}\right\rangle \otimes|\phi\rangle \rightarrow\left|\psi_{2}\right\rangle \otimes|\phi\rangle\right) .
$$

Third, by Theorem 1 we have $P_{E}\left(\left|\psi_{1}\right\rangle \rightarrow\left|\psi_{2}\right\rangle\right)=$ $P_{M}\left(\left|\psi_{1}\right\rangle \rightarrow\left|\psi_{2}\right\rangle\right)$. This together with Eqs. (16) and (17) yields that

$$
P_{M}\left(\left|\psi_{1}\right\rangle \rightarrow\left|\psi_{2}\right\rangle\right)>p \text { iff } p<\min \left\{1, \frac{\alpha_{n}}{\beta_{n}}\right\} .
$$

The condition on right-hand side of Eq. (18) is fulfilled by the assumption. Thus we have $P_{M}\left(\left|\psi_{1}\right\rangle \rightarrow\left|\psi_{2}\right\rangle\right)>p$. By the definition of $P_{M}\left(\left|\psi_{1}\right\rangle \rightarrow\left|\psi_{2}\right\rangle\right)$, there exists a finite positive integer $m$ such that

$$
\left[p_{\max }\left(\left|\psi_{1}\right\rangle^{\otimes m} \rightarrow\left|\psi_{2}\right\rangle^{\otimes m}\right)\right]^{\frac{1}{m}} \geq p .
$$

This completes the proof of the theorem.
It seems to be a challenging problem to characterize when a catalyst $|\phi\rangle$ can be simulated by a finite-copy transformation in the case of $p=\min \left\{1, \frac{\alpha_{n}}{\beta_{n}}\right\}$.

In conclusion, we have examined the relationship between entanglement-assisted transformation and multiple-copy transformation, and have proved that these two ways of manipulation of bipartite pure states are equivalent in the sense they can simulate each other's ability to implement a desired transformation from a given source state to a given target state with the same optimal conversion probability. It would be interesting to obtain a similar equivalence between entanglementassisted transformation and multiple-copy entanglement transformation for pure states shared by three or more parties, and also to extend our results presented here to mixed states.

The authors wish to acknowledge the colleagues in the Quantum Computation and Quantum Information Research Group for many useful discussions. This work was partly supported by the Natural Science Foundation of China (Grant Nos: 60273003, 60433050, 60321002, and 60305005). R. Duan acknowledges the financial support of Tsinghua University (Grant No. 052420003).
[1] C. H. Bennett and G. Brassard, Proceedings of IEEE International Conference on Computers, Systems, and Signal Processing, Bangalore, India, 1984, pp. 175 - 179.

[2] C. H. Bennett and S. J. Wiesner, Phys. Rev. Lett. 69, 2881 (1992).

[3] C. H. Bennett, G. Brassard, C. Crepeau, R. Jozsa, A. Peres, and W. K. Wootters, Phys. Rev. Lett. 70, 1895 (1993).

[4] D. Jonathan and M. B. Plenio, Phys. Rev. Lett. 83, 3566 (1999).

[5] C. H.Bennett, H. J. Bernstein, S. Popescu, and B. Schumacher, Phys. Rev. A 53, 2046 (1996).

[6] M. A. Nielsen, Phys. Rev. Lett. 83, 436 (1999).

[7] G. Vidal, Phys. Rev. Lett. 83, 1046 (1999).

[8] C. H. Bennett, S. Popescu, D. Rohrlich, J. A. Smolin, and A. V. Thapliyal, Phys. Rev. A 63, 012307 (2001).

[9] S. Daftuar and M. Klimesh, Phys. Rev. A 64, 042314
(2001)

[10] Y. Feng, R. Y. Duan, and M. S. Ying, IEEE. Trans. Inf. Theory 51, 1090 (2005).

[11] Y. Feng, R. Y. Duan, and M. S. Ying, Phys. Rev. A 69, 062310 (2004).

[12] J. Eisert and M. Wilkens, Phys. Rev. Lett. 85, 437 (2000).

[13] G. Vidal and J. I. Cirac, Phys. Rev. Lett. 88, 167903 (2002).

[14] S. Bandyopadhyay, V. Roychowdhury, and U. Sen, Phys. Rev. A 65, 052315 (2002).

[15] R. Y. Duan, Y. Feng, X. Li, and M. S. Ying, Phys. Rev. A 71, 062306 (2005); Report No. quant-ph/0312010

[16] R. Y. Duan, Y. Feng, X. Li, and M. S. Ying, Phys. Rev. A 71, 042319 (2005); Report No. quant-ph/0404148

[17] R. Y. Duan, Y. Feng, and M. S. Ying, Report No. quant-ph/0404046 